\title{
Critical sound in fluids and mixtures
}

\author{
R.Folk ${ }^{1}$, G.Moser ${ }^{2}$ \\ 1 Institute for Theoretical Physics, University of Linz, Austria \\ 2 Institute for Physics and Biophysics, University of Salzburg, Austria
}

Received June 26, 1998

The behaviour of sound is explained within the dynamical renormalization group theory. Non-asymptotic effects are due to the deviation of renormalized couplings from their fixed point values. We calculate the temperature and frequency dependence of the sound velocity and absorption near the consolute point and the gas liquid critical point in pure fluids and mixtures. The critical non-asymptotic time scale in mixtures is different from the pure fluid case and set by an effective order parameter Onsager coefficient containing the dynamical parameter related to the enhancement of the thermal conductivity. We discuss the relation to the phenomenological theory of Ferrell and Bhattacharjee for the consolute point and compare with experiments in pure ${ }^{3} \mathrm{He},{ }^{4} \mathrm{He}$ and ${ }^{3} \mathrm{He}-{ }^{4} \mathrm{He}$ mixtures near the plait point.

Key words: critical dynamics, sound propagation, consolute point, plait point, renormalization group theory, transport properties

PACS: $05.70 . J k, 64.60 . H t, 64.70 . F x, 64.70 . J a$

\section{Introduction}

Fluids and mixtures at their critical points, like the gas-liquid critical point (plait point in mixtures) and the demixing (or consolute) point, belong to the same static and dynamic universality class. All static critical effects can be related to the singular behaviour of the Ising model universality class; all dynamic critical effects can be related to the singularities of model $\mathrm{H}$ [1]. This is true for critical effects in the sound propagation too.

Non-asymptotic effects describing the crossover from the non-critical non-universal background to the universal asymptotics depend on the system considered and is different for pure fluids and mixtures. This is in general due to the fact that the fluid parameters entering the physical quantity considered have not reached their asymptotic (fixed point) values. One striking example is the thermal conductivity near the consolute point, which turns out to be finite at the critical point. Its non-universal enhancement is related to the non-asymptotic behaviour of a certain dynamical parameter with fixed point value of zero. Thus the parameter drops out 
of the expression for the thermal conductivity in the asymptotics, but determines its (lower) background value [2]. This enhancement at the consolute point has indeed been observed in 2-butoxy-ethanol-water mixture at the critical concentration $[3,4]$.

In this review we present recent progress in calculating the non-asymptotic crossover functions for the sound velocity and sound attenuation as a function of temperature and frequency (see [5] for pure fluids and [6] for mixtures).

The critical sound propagation in pure fluids and near a consolute point has been described by Ferrell and Bahttacharjee within a phenomenological theory based on a generalization of the specific heat to finite frequencies taking into account the causal and scaling properties of the dynamic functions involved $[7,8]$. An earlier application of the RG theory to critical sound at the consolute point used a reduced model neglecting e.g. the thermal diffusion ratio [9]. Recently Onuki used the bulk viscosity [10] for an intuitive derivation of the critical sound behaviour [11] which is in agreement with our RG calculations in asymptotics and is also valid at the plait point.

It turns out that the non-asymptotic time scale appearing in the frequency dependent sound velocity and attenuation in a mixture is not set by the order parameter Onsager coefficient alone but by an effective one which contains the dynamic parameter responsible for the critical enhancement of the thermal conductivity.

\section{The dynamical equations}

The starting point of the theoretical calculations is a stochastic model fulfilling the constraints. In equilibrium it is described by the usual $\phi^{4}$-model including in a quadratic form the additional dynamic densities and a coupling between the order parameter and the other degrees of freedom. The static functional for a mixture reads (the reduction to the pure fluid model is easily performed)

$$
\begin{aligned}
H=\int \mathrm{d}^{d} x & \left\{\frac{1}{2} \stackrel{o}{\tau} \phi_{0}^{2}(x)+\frac{1}{2}\left(\boldsymbol{\nabla} \phi_{0}(x)\right)^{2}+\frac{1}{2} \vec{q}_{0}^{T}(x) \stackrel{\leftrightarrow}{A} \vec{q}_{0}(x)+\frac{1}{2} a_{j} j^{2}(x)\right. \\
& \left.+\frac{\tilde{u}}{4 !} \phi_{0}^{4}(x)+\frac{1}{2} \stackrel{o}{\vec{\gamma}_{q}^{T}} \vec{q}_{0}(x) \phi_{0}^{2}(x)-\stackrel{o}{h}_{q}^{T} \vec{q}_{0}(x)\right\}
\end{aligned}
$$

where the order parameter $\phi$ is the entropy density in the case of a gas-liquid phase transition and the concentration fluctuation $c$ in the case of the demixing transition. The upright $o$ at the parameters and the subscript ${ }_{0}$ on the densities denote unrenormalized quantities. The static functional has the same structure as in pure liquids [5] but now with two respective secondary densities $\vec{q}_{0}^{T}=\left(\stackrel{o}{q}{ }_{1}, \stackrel{o}{q}_{2}\right)$. As a consequence the parameters

$$
\overleftrightarrow{A}=\left(\begin{array}{ll}
a_{11} & a_{12} \\
a_{12} & a_{22}
\end{array}\right), \quad \stackrel{o}{\gamma}_{q}=\left(\begin{array}{l}
\stackrel{o}{\gamma_{1}} \\
o \\
\gamma_{2}
\end{array}\right), \quad \overrightarrow{\vec{h}}_{q}=\left(\begin{array}{c}
o \\
h_{1} \\
o \\
h_{2}
\end{array}\right)
$$

are matrices and vectors instead of scalars. The momentum density appearing in (1) is also rescaled by the Avogadro number $\boldsymbol{j}=\sqrt{N_{\mathrm{A}}} \triangle \boldsymbol{j}^{\prime}$. The parameter $a_{j}$ follows 
from the expression for the kinetic energy and reads $a_{j}=1 /(R T \rho)$. The coefficients of the matrix $\overleftrightarrow{A}$ are the lowest order contributions to the static two-point vertex functions and therefore do not contain critical effects. They are related to background values of thermodynamic derivatives at the plait and the consolute point respectively.

The dynamical model equations are an extension of model $\mathrm{H}$ [1]

$$
\begin{aligned}
& \frac{\partial \phi_{0}}{\partial t}=\stackrel{o}{\Gamma} \nabla^{2} \frac{\delta H}{\delta \phi_{0}}+\stackrel{o}{\vec{L}_{q}^{T}} \nabla^{2} \frac{\delta H}{\delta \vec{q}_{0}}-\stackrel{o}{g}\left(\nabla \phi_{0}\right)\left(\frac{\delta H}{\delta \boldsymbol{j}_{l}}+\frac{\delta H}{\delta \boldsymbol{j}_{t}}\right)+\Theta_{\phi}, \\
& \frac{\partial \vec{q}_{0}}{\partial t}=\stackrel{o}{\vec{L}_{q}} \nabla^{2} \frac{\delta H}{\delta \phi_{0}}+\stackrel{\stackrel{\leftrightarrow}{\Lambda}}{q} \nabla^{2} \frac{\delta H}{\delta \vec{q}_{0}}-\stackrel{o}{g}\left(\nabla \vec{q}_{0}\right)\left(\frac{\delta H}{\delta \boldsymbol{j}_{l}}+\frac{\delta H}{\delta \boldsymbol{j}_{t}}\right) \\
& -\left(\stackrel{o}{\vec{c}_{q}}+\stackrel{o}{\vec{g}_{l}} \phi_{0}+\stackrel{\stackrel{\leftrightarrow}{g}}{q}_{q} \vec{q}_{0}\right) \nabla \frac{\delta H}{\delta \boldsymbol{j}_{l}}+\vec{\Theta}_{q} \\
& \frac{\partial \boldsymbol{j}_{l}}{\partial t}=\stackrel{o}{\lambda}_{l} \nabla^{2} \frac{\delta H}{\delta \boldsymbol{j}_{l}}-\stackrel{o}{\vec{c}}_{q}^{T} \nabla \frac{\delta H}{\delta \vec{q}_{0}}-\stackrel{o}{\vec{g}}_{l}^{T} \boldsymbol{\nabla}\left(\phi_{0} \frac{\delta H}{\delta \vec{q}_{0}}\right)-\nabla\left(\vec{q}_{0}^{T} \stackrel{\stackrel{\leftrightarrow}{g}}{q}_{q} \frac{\delta H}{\delta \vec{q}_{0}}\right) \\
& +\stackrel{o}{g}(1-\mathcal{T})\left[\left(\boldsymbol{\nabla} \phi_{0}\right) \frac{\delta H}{\delta \phi_{0}}+\left(\boldsymbol{\nabla} \vec{q}_{0}\right)^{T} \frac{\delta H}{\delta \vec{q}_{0}}\right] \\
& -\stackrel{o}{g}(1-\mathcal{T}) \sum_{k}\left[j_{k} \nabla \frac{\delta H}{\delta j_{k}}-\nabla_{k} \boldsymbol{j} \frac{\delta H}{\delta j_{k}}\right]+\boldsymbol{\Theta}_{l}, \\
& \frac{\partial \boldsymbol{j}_{t}}{\partial t}=\stackrel{o}{\lambda}_{t} \nabla^{2} \frac{\delta H}{\delta \boldsymbol{j}_{t}}+\stackrel{o}{g} \mathcal{T}\left[\left(\boldsymbol{\nabla} \phi_{0}\right) \frac{\delta H}{\delta \phi_{0}}+\left(\boldsymbol{\nabla} \vec{q}_{0}\right)^{T} \frac{\delta H}{\delta \vec{q}_{0}}\right] \\
& -\stackrel{o}{g} \mathcal{T} \sum_{k}\left[j_{k} \nabla \frac{\delta H}{\delta j_{k}}-\nabla_{k} \boldsymbol{j} \frac{\delta H}{\delta j_{k}}\right]+\boldsymbol{\Theta}_{t}
\end{aligned}
$$

where $\mathcal{T}$ is the projector to the direction of the transverse momentum density. The static functional $H$ is given by (1). Assuming a Markovian process the fluctuating forces $\left[\Theta_{i}\right]^{T}=\left(\Theta_{\phi}, \vec{\Theta}_{q}^{T}, \Theta_{l}, \Theta_{l}\right)$ fullfill the Einstein relations

$$
\left\langle\left[\Theta_{i}\right](x, t) \otimes\left[\Theta_{j}\right]\left(x^{\prime}, t^{\prime}\right)\right\rangle=2\left[\Lambda_{i j}\right] \delta\left(t-t^{\prime}\right) \delta\left(x-x^{\prime}\right)
$$

with the matrix

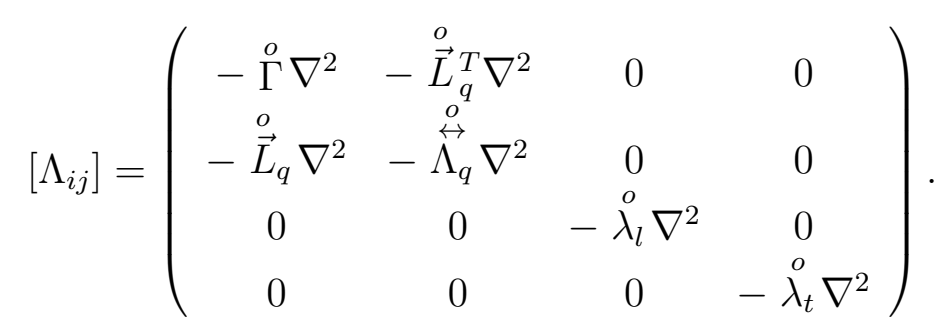

The Onsager coefficient vector and the Onsager coefficient matrix in (3) and (4) are

$$
\vec{L}_{q}=\left(\begin{array}{c}
o \\
L \\
o \\
L_{\phi}
\end{array}\right), \quad \stackrel{o}{\stackrel{\Lambda}{\Lambda}}=\left(\begin{array}{cc}
o & o \\
\mu & L_{12} \\
o & o \\
L_{12} & \lambda
\end{array}\right)
$$


The mode coupling vectors and the mode coupling matrix introduced in (4) and (5) are defined as

$$
\stackrel{o}{\vec{c}_{q}}=\left(\begin{array}{c}
0 \\
o \\
c
\end{array}\right), \quad \stackrel{o}{\vec{g}}_{l}=\left(\begin{array}{c}
0 \\
o \\
g_{l}
\end{array}\right), \quad \stackrel{\stackrel{o}{g}}{q}=\left(\begin{array}{cc}
0 & 0 \\
0 & o \\
g
\end{array}\right)
$$

with the parameters $\stackrel{o}{c}=R T \rho, \stackrel{o}{g}=R T / \sqrt{N_{\mathrm{A}}}$ and $\stackrel{o}{g}=R T Q_{2} / \sqrt{N_{\mathrm{A}}}$ with $Q_{2}$ given by thermodynamic derivatives [6]. The Onsager coefficients in the momentum density equations (5) and (6) are related to the background values of the shear viscosity $\bar{\eta}^{(0)}$ and the bulk viscosity $\zeta^{(0)}$ by $\stackrel{o}{\lambda}_{l}=R T\left(\zeta^{(0)}+\frac{4}{3} \bar{\eta}^{(0)}\right)$ and $\stackrel{o}{\lambda}_{t}=R T \bar{\eta}^{(0)}$.

In hydrodynamics only three transport coefficients, which are the thermal conductivity $\kappa_{\mathrm{T}}$, the thermal diffusion coefficient $k_{\mathrm{T}}$ and the mass diffusion coefficient $D$, appear in the equations for the entropy and the concentration. The hydrodynamic equation for the mass density is the continuity equation, which does not involve any dissipation. As a consequence only three $(\stackrel{o}{\Gamma}, \stackrel{o}{L}$ and $\stackrel{o}{\mu})$ of the six Onsager coefficients in equations (3)-(6) are independent. The remaining three coefficients are determined by

$$
\stackrel{o}{L}_{\phi}=-Q_{2} \stackrel{o}{\Gamma}, \quad \stackrel{o}{\lambda}=Q_{2}^{2} \stackrel{o}{\Gamma}, \quad \stackrel{o}{L}_{12}=-Q_{2} \stackrel{o}{L} .
$$

Consequently the fluctuating forces in (7) are not independent. Nevertheless a dynamic functional analogue to [12] may be derived [5].

We now apply the renormalization group procedure in its field theoretic version (dimensional regularization, minimal subtraction) to this dynamic functional. Comparing the dynamic vertex functions of the model in the hydrodynamic limit with the results from the usual hydrodynamic equations for mixtures [13] leads to expressions for the transport coefficients. Comparing one of them (e.g. the shear viscosity) allows determinating the non-universal background parameters entering the expression of the transport coefficients. In this way all dynamic parameters are fixed and predictions become possible.

\section{Sound velocity and absorption}

By the above mentioned procedure we also find the sound velocity $c_{\mathrm{s}}$ and the sound attenuation $\alpha_{\mathrm{s}}$ at finite frequency $\omega$ and temperature distance $t=\left(T-T_{\mathrm{c}}\right) / T_{\mathrm{c}}$. Both expressions are obtained from the complex sound velocity $\mathcal{C}_{\mathrm{s}}$

$$
c_{\mathrm{s}}^{2}(t, \omega)=\Re\left[\mathcal{C}_{\mathrm{s}}^{2}(t, \omega)\right] \quad \alpha_{\mathrm{s}}(t, \omega)=-\frac{\omega \Im\left[\mathcal{C}_{\mathrm{s}}^{2}(t, \omega)\right]}{2 c_{\mathrm{s}}^{3}(t, \omega)},
$$

where we have neglected subleading and constant background terms restoring the complete hydrodynamic result in the background. It is convenient to eliminate one $\gamma$-coupling in (1) by introducing rotated secondary densities; then only one of them is coupled to the order parameter. After this transformation the structure of the complex sound velocity $\mathcal{C}_{\mathrm{s}}$ turns out to be the same for all critical points in pure 
liquids and mixtures. It agrees in its structure with [11], however our expression contains the non-asymptotic crossover effects. The complex sound velocity reads

$$
\mathcal{C}_{\mathrm{s}}^{2}(t, \omega)=c_{\mathrm{s}}^{2}(0,0)+\left\{c_{\mathrm{s}}^{2}(\bar{t}, 0)-c_{\mathrm{s}}^{2}(0,0)\right\} \frac{1+\gamma^{2}(\bar{t}) F_{+}^{(\mathrm{s})}(u(\bar{t}))}{1+\gamma^{2}(\bar{t}) F_{+}(v(t, \bar{t}), w(\bar{t}))}
$$

This expression reduces at zero frequency to the real static sound velocity $c_{\mathbf{S}}^{2}(t, 0)$, which for both phase transitions (gas-liquid and demixing) is given by $c_{\mathrm{s}}^{2}(t, 0)=$ $\left(\frac{\partial P}{\partial \rho}\right)_{\sigma, c}$. For mixtures the value of the sound velocity at $T_{\mathrm{c}}(t=0)$ is nonzero. The total attenuation reduces to its non-critical background value, thus the above expression for the attenuation is zero.

At finite frequency the fraction on the right hand side of equation 13 becomes different from one, since the dynamic function $F_{+}$becomes different from its static counterpart $F_{+}^{(s)}$. Moreover the effective temperature $\bar{t}=\bar{t}(t, \omega)$, resulting from a matching condition defined below, becomes different from $t$. Now in the full expression the static coupling $\gamma^{2}(\bar{t})$ enters.

The static velocity $c_{\mathrm{s}}$ may be expressed by thermodynamic derivatives parallel to the second order phase transition line with negligible temperature dependence in the critical region and the inverse of a weak diverging $\left(\sim t^{-\alpha}\right)$ susceptibility. At the consolute point this weak diverging susceptibility is the specific heat at constant pressure and concentration [14],

$$
\begin{aligned}
c_{\mathrm{s}}^{2}(0,0) & =\frac{T}{\rho^{2}}\left(\frac{\partial P}{\partial T}\right)_{c, T_{\mathrm{c}}}^{2} C_{c, T_{\mathrm{c}}}^{-1}, \\
c_{\mathrm{s}}^{2}(\bar{t}, 0)-c_{\mathrm{s}}^{2}(0,0) & =\frac{c_{\mathrm{s}}^{2}(0,0)}{\left(\frac{\partial T}{\partial \sigma}\right)_{c, T_{\mathrm{c}}}^{2} C_{c, T_{\mathrm{c}}} / T-\left(\frac{\partial T}{\partial \sigma}\right)_{c, P}}\left(\frac{\partial T}{\partial \sigma}\right)_{c, P}
\end{aligned}
$$

with $C_{c, T_{\mathrm{c}}}=T\left(\frac{\partial \sigma}{\partial T}\right)_{c, T_{\mathrm{c}}}+\frac{T}{\rho^{2}}\left(\frac{\partial P}{\partial T}\right)_{c, T_{\mathrm{c}}}\left(\frac{\partial \rho}{\partial T}\right)_{c, T_{\mathrm{c}}}$. From these expressions we can derive the coupling constant used in the ansatz of the phenomenological theory of Ferrell and Bhattacharjee $[15,8]$ for pure fluids and mixtures near the consolute point.

For the plait point the weak diverging susceptibility is the concentration susceptibility at constant pressure and entropy,

$$
\begin{aligned}
c_{\mathrm{s}}^{2}(0,0) & =\frac{1}{\rho^{2}}\left(\frac{\partial P}{\partial \Delta}\right)_{\sigma, T_{\mathrm{c}}}^{2} \chi_{\sigma, T_{\mathrm{c}}}^{-1}, \\
c_{\mathrm{s}}^{2}(\bar{t}, 0)-c_{\mathrm{s}}^{2}(0,0) & =\frac{c_{\mathrm{s}}^{2}(0,0)}{\left(\frac{\partial \Delta}{\partial c}\right)_{\sigma, T_{\mathrm{c}}}^{2} \chi_{\sigma, T_{\mathrm{c}}}-\left(\frac{\partial \Delta}{\partial c}\right)_{\sigma, P}}\left(\frac{\partial \Delta}{\partial c}\right)_{\sigma, P}
\end{aligned}
$$

with $\chi_{\sigma, T_{\mathrm{c}}}=\left(\frac{\partial c}{\partial \Delta}\right)_{\sigma, T_{\mathrm{c}}}+\frac{1}{\rho^{2}}\left(\frac{\partial P}{\partial \Delta}\right)_{\sigma, T_{\mathrm{c}}}\left(\frac{\partial \rho}{\partial \Delta}\right)_{\sigma, T_{\mathrm{c}}}$. Using thermodynamic relations one may rewrite these expressions involving other 'weak' susceptibilities on the right hand side. The non-universal values of the static quantities, both of $c_{\mathrm{s}}(0,0)$ (large for the consolute point, small for the plait point and zero for the pure liquid) and of the 


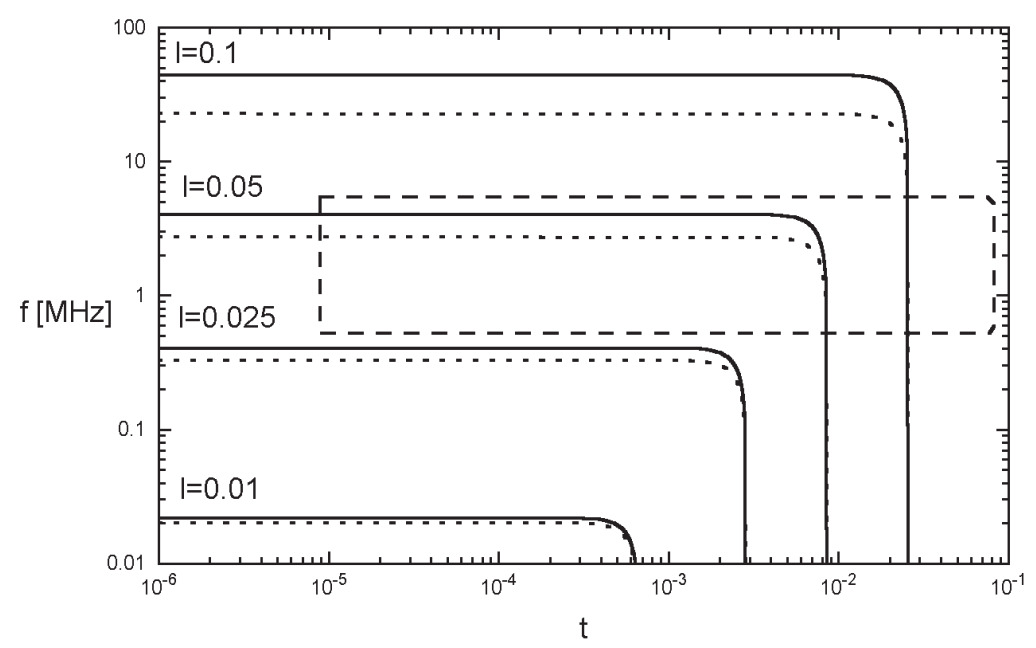

Figure 1. Curves of 'equal distance from criticallity' in the frequency-temperature plane. Full lines calculated from the temperature dependence of $\Gamma(t)$ shown in figure $2 \mathrm{~b}$, dotted lines calculated from the asymptotic behaviour $\Gamma(t)$

weak diverging susceptibility (dominated by its background term for the consolute point, pure liquid like behaviour for the plait point) might be quite different and in consequence lead to a different frequency dependence of the sound velocity and absorption.

This different behaviour of the 'weak' susceptibility has also consequences for the static coupling because $\gamma$ is related to the logarithmic derivatives of the singular part of the sound velocity and may be approximated by

$$
\gamma^{2}(\bar{t}) \approx-\frac{2}{\nu} \frac{\mathrm{d} \ln \left\{c_{\mathrm{s}}^{2}(\bar{t}, 0)-c_{\mathrm{s}}^{2}(0,0)\right\}}{\mathrm{d} \ln \bar{t}}
$$

In the asymptotic limit it reaches its fixed point value $\gamma^{\star 2}=2 \frac{\alpha}{\nu}$ whereas in the background it goes to zero. Depending on the sign of the Wegner correction in the weak susceptibility $\gamma$ reaches zero monotonically (at consolute points) or has a maximum larger than its critical value (gas-liquid critical points).

The function $F_{+}$is an amplitude function, related to the so-called 'frequency dependent specific heat', which may also be calculated within model H' and reads in one loop order

$$
F_{+}(v, w)=-\frac{1}{4}\left\{\frac{v^{2}}{v_{+} v_{-}} \ln v+\frac{1}{v_{+}-v_{-}}\left[\frac{v_{-}^{2}}{v_{+}} \ln v_{-}-\frac{v_{+}^{2}}{v_{-}} \ln v_{+}\right]\right\}
$$

with $v_{ \pm}=\frac{v}{2} \pm \sqrt{\left(\frac{v}{2}\right)^{2}+\mathrm{i} \bar{w}}, v(t, \bar{t})=\xi^{-2}(t) / \xi^{-2}(\bar{t})$ and $\bar{w}(\bar{t})=\omega /\left(2 \Gamma_{\text {eff }}(\bar{t}) \xi^{-4}(\bar{t})\right)$. The dependence of the function on its variables is the same for pure fluids and 

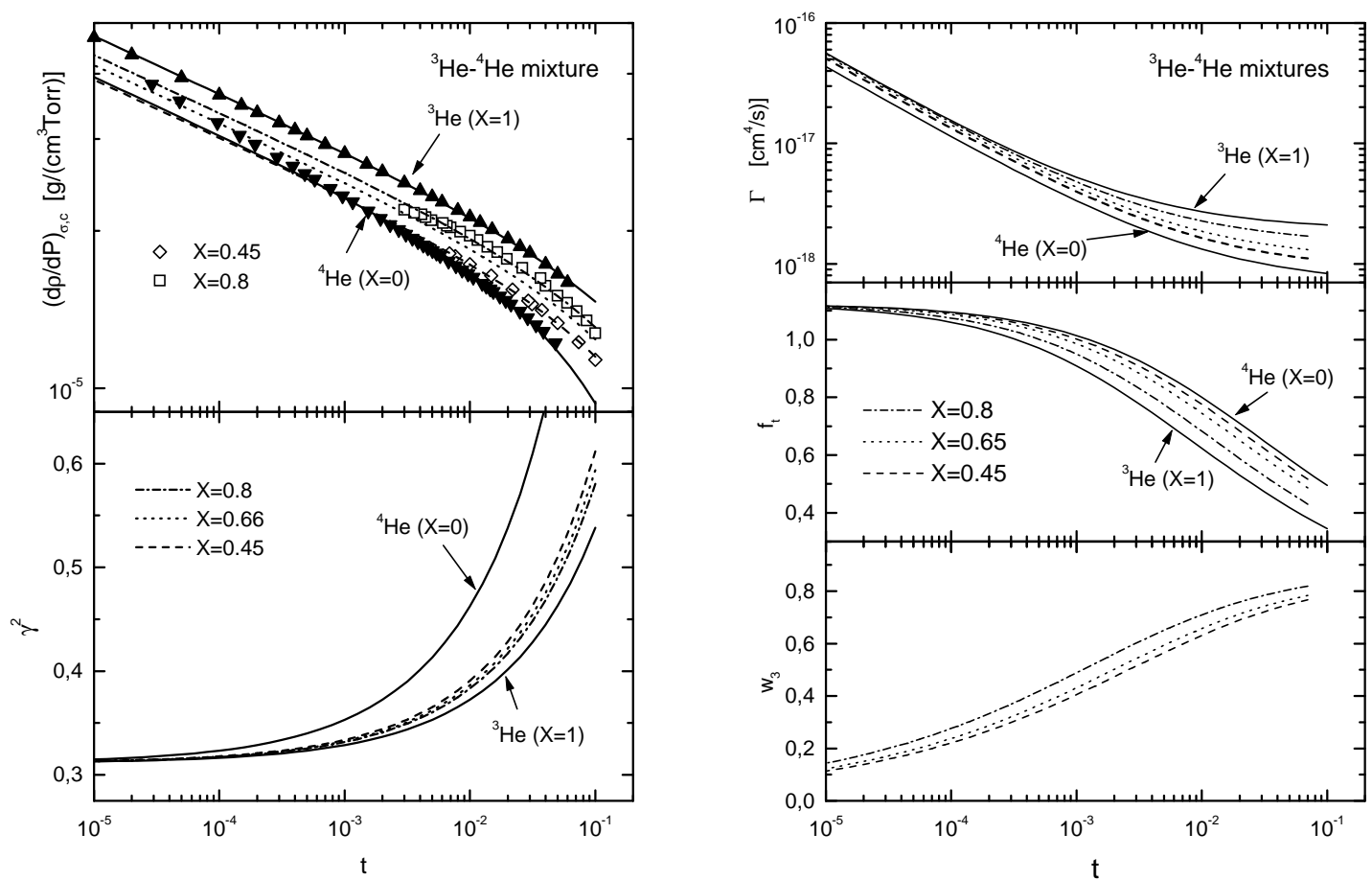

Figure 2. Crossover behaviour of (a) the static coupling $\gamma(t)$ between the order parameter and sound calculated from the adiabatic compressibility and (b) for the dynamic parameters for pure ${ }^{3} \mathrm{He}$ and ${ }^{4} \mathrm{He}$ and mixtures (from [6]).

mixtures. This is a manifestation of universality for all phase transitions considered here. The only difference between pure fluids and mixtures lies in the definition of the effective Onsager coefficient $\bar{\Gamma}$ in the non-asymptotic region.

The temperature distance $t$ and the frequency $\omega$ enter explicitly but also via the effective temperature distance $\bar{t}$, which is finite at finite frequency in the limit $t=0$ and which is found from the matching condition $t^{8 \nu}+\left(2 \xi_{0}^{4} \omega / \Gamma_{\text {eff }}(\bar{t})\right)^{2}=\bar{t}^{8 \nu}$. This effective temperature appears also in $c_{\mathrm{s}}^{2}(\bar{t}, 0)-c_{\mathrm{s}}^{2}(0,0)$ and leads to the respective asymptotic power law behaviour in frequency or temperature. Due to the matching condition our expression for $F_{+}$remains finite in the asymptotic limits $(t \rightarrow 0$ and or $\omega \rightarrow 0)$. No exponentiation is performed. Expanding the static part down to the denominator one gets agreement with $[16,17]$. Comparing with the result in [11] in the asymptotic region shows that Onuki's function $\mathcal{F}$ is represented in our theory by the second part of equation (13) divided by $c_{\mathrm{s}}^{2}(t, 0)-c_{\mathrm{S}}^{2}(0,0)$. The quantitative differences between the asymptotic limit of our theory and Onuki's results for pure fluids have been presented recently [18] in a reconsideration of the sound data of ${ }^{3} \mathrm{He}$ and ${ }^{4} \mathrm{He}$.

The effective OC of the order parameter, which appears in the frequency variable $\bar{w}$ and the matching condition, contains for the mixtures the dynamic param- 

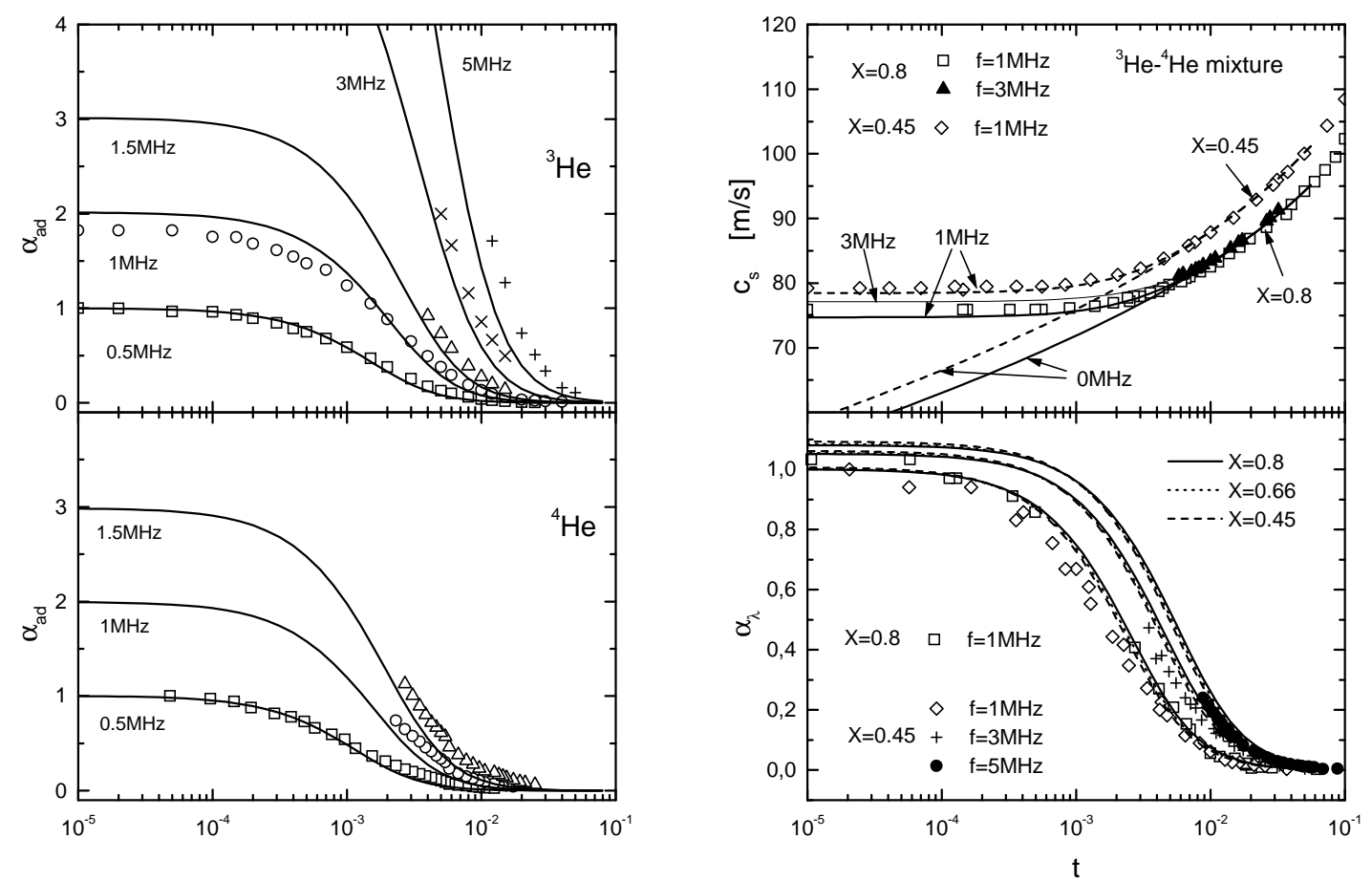

Figure 3. Sound attenuation for (a) pure He (from [5]) and sound velocity and sound attenuation for mixtures (from [6]).

eter $w, \Gamma_{\text {eff }}=\Gamma(1-w)$. As has been mentioned in the introduction this parameter determines the enhancement of the thermal conductivity (it is genuine of dynamical origin; another more complicated and dominating effect determines the critical enhancement $[19,6]$ near the plait point). Since the fixed point value of $w^{\star}=0$ asymptotically there is no difference in the asymptotic region to the the pure fluid case. In fact the asymptotic behaviour is completely determined by the pure fluid exponents and scaling functions. Differences between pure fluids and mixtures arise only because of the nonzero value of the corresponding sound velocity at $T_{\mathrm{c}}$ in the mixtures. Observing this, the scaling properties of the attenuation agree with those mentioned in [20] for the consolute point.

\section{Experimental comparison}

We are now ready to compare with experimental data ${ }^{3} \mathrm{He}-{ }^{4} \mathrm{He}$ mixtures and the pure components at the gas-liquid critical point. For ${ }^{3} \mathrm{He}-{ }^{4} \mathrm{He}$ mixtures the finite value of the sound velocity at $T_{\mathrm{c}}$ is not observable. Therefore all formulas reduce within the experimental region to the pure fluid case. Moreover the static singular part of the sound velocity $c_{\mathrm{s}}^{2}(t)$ behaves as $t^{\alpha}\left(1+g t^{0.5}\right)$ with a positive amplitude $g$ of the Wegner correction leading to the temperature dependence of the coupling 


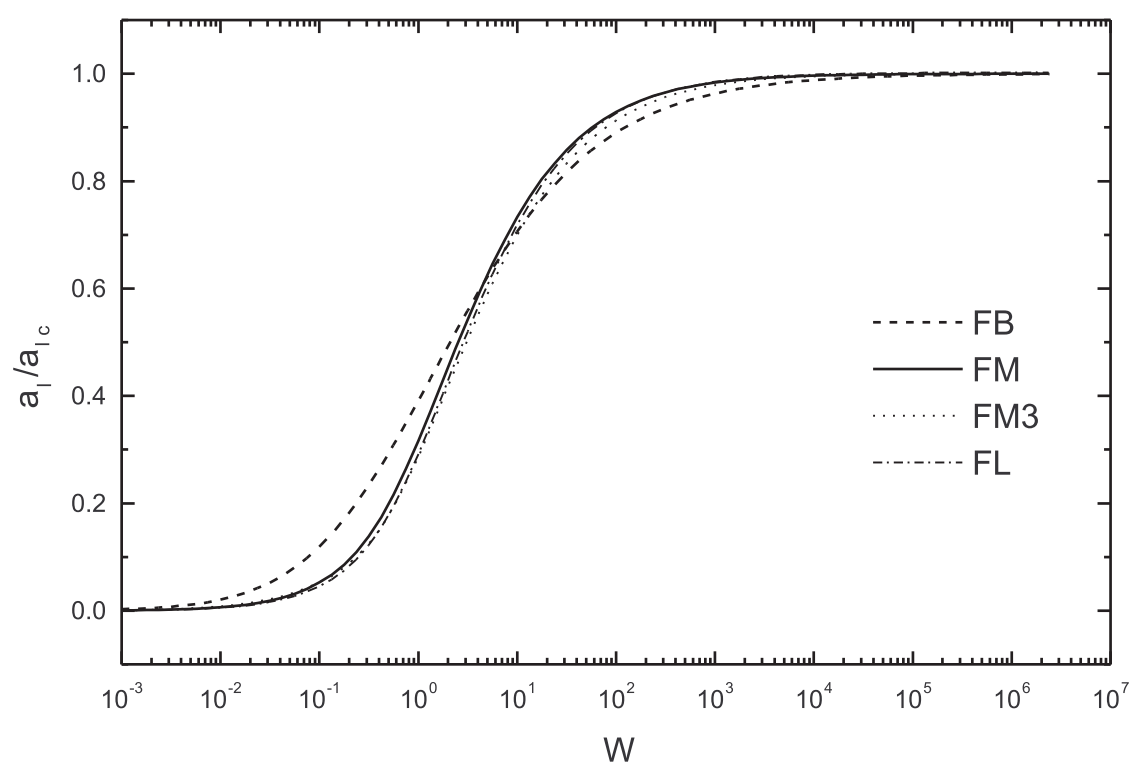

Figure 4. Comparison of different scaling functions for the normalized sound attenuation in one wavelength (from [6]) FB: Ferrell Bhattacharjees empirical function, FM, FM3: our result obtained from an $\varepsilon$-expansion and a $d=3$ calculation, FL: result for pure fluids.

$\gamma$ shown in figure 2a. Accordingly a maximum is to be expected but not seen in the temperature region of the experiments. Both dynamic parameters $w(t)$ and $\Gamma(t)$ can be determined within the dynamical equations of model H', uncoupled to the sound degrees of freedom. We take the theoretical shear viscosity $[5,6]$ at zero frequency and compare it with the experimental shear viscosity [21] in order to find the background values of $w, f$ and $\Gamma$. The resulting temperature dependence of the order parameter Onsager coefficient, the mode coupling $f$ and the parameter $w$ is shown in figure $2 \mathrm{~b}$. The remaining input in the calculation of the frequency dependence of the sound velocity and sound absorption is the sound velocity at zero frequency. It may be either taken from a sound velocity measurement or calculated from thermodynamic relations. Putting everything together and inserting into (12) and (13) we compare with the data for the attenuation in pure He $[22,23]$ in figure 3 a and with the sound velocity and attenuation in mixtures [24] in figure 3b. Thereby we have normalized the attenuation at $t=10^{-5}$ and $1 \mathrm{MHz}$. The differences between the values at $t=10^{-5}$ of the $1 \mathrm{MHz}$ and $3 \mathrm{MHz}$ data are due to the non-asymptotic behaviour of the static coupling $\gamma$, which has not reached its fixed point value for these frequencies.

Concerning the consolute point we compare the result of our theory for the normalized sound attenuation in one wave length with the phenomenological theory of Ferrell and Bhattacharjee in figure 4. Quantitative differences appear only to the empirical expression. Due to the specific expression for $\alpha_{\lambda}$ and the approximation allowed in the experimental region one obtains almost the same result for this quan- 
tity as suggested in [25]. To some extent one may include non-asymptotic effects in "scaling functions" see e.g. [5].

Ackowledgement: We acknowledge support by the Fonds zur Foerderung der Wissenschaftlichen Forschung under project 12422-TPH.

\section{References}

1. Siggia E.D., Halperin B.I., Hohenberg P.C. Renormalization-group treatment of the critical dynamics of the binary-fluid and gas-liquid transition. // Phys. Rev. B, 1976, vol. 13, No. 5, p. 2110-2132.

2. Folk R., Moser G. Nonasymptotic transport properties in fluids and mixtures near a critical point. // Int. J. Thermophys., 1998, vol. 19, No. 4, p. 1003-1017.

3. Mensah-Brown H., Wakeham W.A. Thermal conductivity of water and 2-nbutoxyethanol and their mixtures in the temperature range 305-350 at pressures up to $150 \mathrm{MPa}$. // Int. J. Thermophys., 1995, vol. 16, No. 1, p. 237-244.

4. Zielensny A., Schmitz J., Limberg S, Aizpiri A.G., Fusenig S., Woerman D. Viscosity and diffusivity of a binary liquid mixture of critical composition: Study of the system 2-butoxyethanol/water. // Int. J. Thermophys., 1994, vol. 15, No. 1, p. 67-94.

5. Folk R., Moser G. Frequency-dependent shear viscosity, sound velocity and sound attenuation near the critical point in liquids. I. Theoretical results. // Phys. Rev. E, 1998, vol. 57, No. 1, p. 683-704; II. Comparison with experiment.//Phys. Rev. E, 1998, vol. 57, No. 1, p. 705-719.

6. Folk R., Moser G. Critical dynamics in mixtures. // Phys. Rev. E, 1998, vol. 58, No. 5, p. 6246-6274; Critical sound propagation in mixtures. // Europhys. Lett., 1998, vol. 41, No. 2, p. 177-182.

7. Ferrell R.A., Bhattacharjee J.K. Dynamic universality and the critical sound velocity in a binary liquid. // Phys. Rev. B, 1981, vol. 24, No. 7, p. 4095-4097.

8. Ferrell R.A., Bhattacharjee J.K. Dynamic scaling theory of the critical attenuation and dispersion of sound in a classical fluid: The binary liquid. // Phys. Rev. A, 1985, vol. 31, No. 3, p. 1788-1809.

9. Kroll D. M., Ruhland J.M. Sound propagation in critical binary mixtures. // Phys. Rev. A, 1981, vol. 23, No. 1, p. 371-374.

10. Kawasaki K. // Phys. Rev. A, 1970, vol. 1, p. 1750.

11. Onuki A. J. Bulk viscosity near the critical point. // Phys. Soc. Jap., 1997, vol. 66, No. 3, p. 511-513.

12. Bausch R., Janssen H.K., Wagner H. Renormalized field theory of critical dynamics. // Z. Phys. B, 1976, vol. 24, p. 113-127.

13. Landau L.D., Lifshitz E.M. Fluid Mechanics. 2nd ed. New York, Pergamon, 1987.

14. Ferrell R.A. Dynamic-scaling theory of critical ultrasonic attenuation in binary liquids. // Int. J. Thermophys., 1989, vol. 10, No. 2, p. 369-379.

15. Bhattacharjee J.K., Ferrell R.A. General dynamic scaling theory of critical ultrasonic attenuation and dispersion. // Phys. Lett. A, 1981, vol. 86, No. 2, p. 109-112 ; Critical ultrasonic and hypersonic attenuation for the liquid-gas phase transition. // Phys. Lett. A, 1982, vol. 88, No. 2, p. 77-80.

16. Kroll D.M., Ruhland J.M. Renormalization group calculation of sound attenuation and dispersion near the liquid-gas critical point. // Phys. Lett. A, 1980, vol. 80, No. 1, 
p. $45-48$.

17. Onuki A. Dynamic equations and bulk viscosity near the gas-liquid critical point. // Phys. Rev. E, 1997, vol. 55, No. 1, p. 403-420.

18. Kogan A., Meyer H. Sound propagation in ${ }^{3} \mathrm{He}$ and ${ }^{4} \mathrm{He}$ above the liquid-vapor critical point. // J. Low Temp. Phys., 1998, vol. 10, No. 3/4, p. 899-918.

19. Folk R., Moser G. Critcal dynamics near the liquid-vapour transition in mixtures. // Europhys. Lett., 1993, vol. 24, No. 7, p. 533-538.

20. Dengler R., Schwabl F. Universal ratios of critical sound attenuation. // Z. Physik B, 1987, vol. 69, p. 327-338. RGT predicts an asymptotic frequency dependence of the sound absorption in one wave length at $T_{\mathrm{c}}, \alpha_{\lambda} \sim \omega^{\frac{\alpha}{z \nu}}$, whereas in the approximation of Ferrell and Bhattacharjee one obtains $\alpha_{\lambda} \sim \omega^{-\frac{\alpha}{z \nu}}$.

21. Wang S., Meyer H. Transport properties of helium near the liquid-vapor critical point. V. The shear viscosity of ${ }^{3} \mathrm{He}^{-}{ }^{4} \mathrm{He}$ mixtures. // J. Low Temp. Phys., 1987, vol. 69, No. 5/6, p. 377-390.

22. Roe D.B., Wallace B.A., Meyer H. Ultrasonic dispersion and attenuation near the liquid-gas critical point in ${ }^{4}$ He. // J. Low Temp. Phys., 1974, vol. 16, No. 1/2, p. 5172 .

23. Roe D.B., Meyer H. Ultrasonic dispersion and attenuation near the liquid-gas critical point in ${ }^{3}$ He. // J. Low Temp. Phys., 1978, vol. 30, No. 1/2, p. 91-115.

24. Doiron T., Gestrich D., Meyer H. Sound propagation in ${ }^{3} \mathrm{He}-{ }^{4} \mathrm{He}$ mixtures near the liquid-vapor critical point. // Phys. Rev. B, 1980, vol. 22, No. 7, p. 3202-3206.

25. Bhattacharjee J.K., Ferrell R.A. Universality in the critical dynamics of fluids: ultasonic attenuation. // Physica A, 1998, vol. 250, p. 83-90. 


\title{
Критичний звук у рідинах та сумішах
}

\author{
Р. Фольк ${ }^{1}$, Г. Мозер ${ }^{2}$
}

1 Інститут теоретичної фізики, університет м.Лінц, Лінц, Австрія

2 Інститут фізики і біофізики, університет м.Зальцбург, Зальцбург, Австрія

Отримано 26 червня 1998 р.

У теорії динамічної ренормалізаційної групи пропонується пояснення особливостей поведінки критичного звуку. Показано, що неасимптотичні ефекти обумовлені відхиленням ренормованих параметрів взаємодії від їх фіксованих значень. Для ряду чистих рідин та сумішей розраховані температурна й частотна залежності швидкості звуку і коефіцієнта поглинання поблизу точки розшарування і критичної точки рідина-газ. Знайдено, що неасимптотичний критичний часовий масштаб у сумішах відрізняється від того, що отримується для випадку чистих плинів, і обумовлено це зростанням ролі термопровідності. Обговорюється зв'язок з феноменологічною теорією Феррелла і Бхаттачарі для точки розшарування та проводиться порівняння з експериментами для чистих плинів ${ }^{3} \mathrm{He}$ i ${ }^{4} \mathrm{He}$ та їх сумішей поблизу критичної точки.

Ключові слова: критична динаміка, поширення звуку, точка розшарування, критична точка газ-рідина, теорія ренормгрупи, властивості переносу

PACS: $05.70 . J k, 64.60 . H t, 64.70 . F x, 64.70 . J a$ 